\title{
Acid-Base Behavior in 50-Percent Aqueous Methanol: Thermodynamics of the Dissociation of Protonated Tris(hydroxymethyl)aminomethane and Nature of the Solvent Effect ${ }^{*}$
}

\author{
Maurice Woodhead ${ }^{1}$ Maya Paabo, R. A. Robinson, and Roger G. Bates
}

(February 16, 1965)

\begin{abstract}
Electromotive-force methods and cells without liquid junction have been utilized to determine the acidic dissociation constant of protonated tris(hydroxymethyl)aminomethane [2-amino-2-(hydroxymethyl)-1, 3-propanediol] in $50 \mathrm{wt}$ percent methanol solvent at seven temperatures from 10 to $40{ }^{\circ} \mathrm{C}$. The change of the dissociation constant with temperature has been used to calculate the changes of enthalpy, entropy, and heat capacity when the dissociation takes place in the standard state. Comparisons with earlier measurements in the aqueous medium reveal no great differences in the enthalpy and entropy, suggesting that water participates in preference to methanol in the protolytic reaction even in 50-percent methanol. It is shown that electrostatic considerations alone are unable to explain the solvent effect on the dissociation energy, and a substantial "basicity effect" is indicated. The activity-coefficient term for the amine hydrochloride in equimolal amine-salt buffers has been evaluated and compared with similar data in the water solvent.
\end{abstract}

\section{Introduction}

Acid-base studies of organic compounds only slightly soluble in water are sometimes conveniently made in a solvent consisting of equal parts by weight of water and methanol. In order to facilitate the determination of $p \mathrm{H}$ and $p K$ in 50-percent methanol, an operational $p \mathrm{H}$ scale has recently been established for this solvent mixture $[1]^{2}$. The standard scale, defined in a manner consistent with thermodynamic dissociation constants and activity coefficients in 50-percent methanol, is fixed by three suitable reference solutions, namely an acetate buffer, a phosphate buffer, and a solution of sodium hydrogen succinate.

As a part of the earlier work, the dissociation constants of acetic acid and dihydrogen phosphate ion in 50-percent methanol were determined from 10 to $40{ }^{\circ} \mathrm{C}$. These two acids are of charge types $\mathrm{A}^{0} \mathrm{~B}^{-}$ and $\mathrm{A}^{-} \mathrm{B}^{=}$, respectively. Here $\mathrm{A}$ and $\mathrm{B}$ refer respectively to the acid and its conjugate base. The results for the enthalpy and entropy of ionization in 50percent methanol were of interest for their bearing on the relation between charge type and solvent effect.

*Presented before the Division of Analytical Chemistry at the 149th National meeting of the American Chemical Society, Detroit, Mich., on April 7, 1965.

${ }^{1}$ Guest worker, on leave from Makerere University College, Kampala, Uganda.

${ }^{2}$ Figures in brackets indicate the literature references at the end of this paper.
The dissociation constant of an acid of charge type $\mathrm{A}^{+} \mathrm{B}^{0}$, namely the acid conjugate to the weak uncharged base 2-amino-2-(hydroxymethyl)-1, 3-propanediol or tris(hydroxymethyl)aminomethane, ${ }^{3}$ has been studied in 50-percent methanol from 10 to $40{ }^{\circ} \mathrm{C}$. The associated thermodynamic quantities have been derived. Buffer solutions composed of this primary amine and its salt have proved extraordinarily useful for $p \mathrm{H}$ control in biological systems [2].

\section{Experimental Methods}

\subsection{Materials}

An aqueous solution of twice-distilled hydrochloric acid was used as a primary standard. Its molality was determined by gravimetric chloride determination; the standard deviation derived from three determinations was 0.01 percent. The purity of four lots of crystalline tris(hydroxymethyl)aminomethane (obtained from commercial sources) was found to be $99.92,99.94,99.94$, and 100.22 percent by titration with the standard solution of hydrochloric acid. Weight burets were used, and each sample was adjusted to the theoretical equivalence point $(p \mathrm{H} 4.54$ in a $0.1 M$ solution of the neutralized base) with the aid of glass-electrode measurements.

${ }^{3}$ For brevity, this base will sometimes be referred to as "tris" and the corresponding ${ }^{3}$ For brevity, this base will sometim
hydrochloride as "tris hydrochloride." 
For convenience, many of the cell solutions were. prepared from a commercial grade of tris hydrochloride together with the free base. The emf of cells containing buffer solutions prepared in this way was compared with solutions of identical nominal molalities prepared from tris and the standard solution of hydrochloric acid. Three buffer concentrations spanning the range covered in the study were compared in this way. The difference in emf was found to be $0.39 \mathrm{mV}$ with a standard deviation of $0.05 \mathrm{mV}$. The cells prepared with the tris hydrochloride gave the higher emf. The recorded emf for cells prepared in this way was adjusted to correspond to that obtainable with pure tris (assay 100\%) and the standard solution of hydrochloric acid.

On examination, the tris hydrochloride obtained commercially was found to be very close to stoichiometric neutrality; measurements of $p \mathrm{H}$ and buffer capacity showed that the product contained no appreciable excess of either the base or of hydrochloric acid. Analysis for chloride by gravimetry showed, however, that the acidic and basic components, while present in equivalent amounts, were both present in lower quantity than expected (about 99.1 percent of theoretical). Furthermore, samples of the salt prepared by the authors by neutralizing the pure tris with hydrochloric acid were also found to assay from 99.1 to 99.6 percent.

The inert impurity in the commercial product, thought to be water by the manufacturer, could not be identified. ${ }^{4}$ Drying at temperatures low enough to preclude decomposition of the salt did not materially increase the assay value. ${ }^{5}$ Although drying brought about some improvement in the results of the carbon and hydrogen analysis, the figures were inconclusive:

\begin{tabular}{|c|c|c|}
\hline & $\begin{array}{l}\text { Carbon, } \\
\text { percent }\end{array}$ & $\begin{array}{c}\text { Hydrogen, } \\
\text { percent }\end{array}$ \\
\hline Commercial sample................. & 30.81 & 7.83 \\
\hline Commercial sample, after drying............... & $\begin{array}{l}30.60 \\
30.60\end{array}$ & $\begin{array}{l}7.69 \\
7.84\end{array}$ \\
\hline Theoretical....... & 30.49 & 7.67 \\
\hline
\end{tabular}

The supposition that the impurity was electrochemically inert was confirmed by a comparison of the correction to the emf observed $(0.39 \mathrm{mV})$ with that to be expected from the known assay $(0.46 \mathrm{mV})$. The difference corresponds to only 0.001 unit in $p K$.

The methanol with which the solvents were prepared was "Spectro Grade," of the same quality as that used in the earlier studies in $50 \mathrm{wt}$ percent methanol solvents $[1,4]$.

${ }^{4}$ Datta, Grzybowski, and Wilson [3] reported a similar low chloride assay for the samples of tris hydrochloride prepared for their study of the dissociation of tris in aqueous solution. ${ }^{5}$ Analysis of the dried sample by gas chromatography and by Karl Fischer methods indicated the presence of 0.1 to 0.3 percent of water. The authors are indebted to R. J. Hall for these results and for the elemental analysis.

\subsection{Procedures}

In general, the experimental procedures followed those used in determining the dissociation constant of tris in water [5]. Electromotive force measurements of the cell

$\mathrm{Pt} ; \mathrm{H}_{2}(\mathrm{~g}, 1 \mathrm{~atm})$, tris $\cdot \mathrm{HCl}\left(m_{1}\right), \operatorname{tris}\left(m_{2}\right)$ in $50 \mathrm{wt}$ percent methanol, AgCl; Ag

where $m_{1}$ and $m_{2}$ are molalities, were used. Thirtyfour solutions, all with buffer ratio close to unity, were studied. When tris hydrochloride was used, a stock buffer solution was prepared by weighing the free base, the salt, and water. The remainder of the solutions were prepared by dissolving a known weight of tris in a known weight of standard hydrochloric acid. The necessary weight of methanol was then added, together with more water, to achieve the desired solvent composition.

Each cell contained one hydrogen electrode and one silver-silver chloride electrode. The emf measurements at the seven temperatures were made in a variety of sequences. A complete series required from two to three days. The solubility of silver chloride in a methanol-water solution of tris was not determined, as it had been found insufficient to require corrections in aqueous solutions [5]. Methanol is presumed to lower the solubility still further.

\subsection{Results}

The emf data are summarized in table 1. Corrections have been made to the reference hydrogen partial pressure of $1 \mathrm{~atm}$ with the aid of the recorded barometric pressure and the known total vapor pressure of the methanol-water solvent at the temperature in question [4]. The emf recorded for those solutions prepared by weighing tris and the solid hydrochloride was further corrected for the known deficiency of ionized chloride in these solutions.

\section{Calculation of the Dissociation Constant}

The calculation of $p K$, where $K$ is the acidic dissociation constant of 2-ammonium-2-(hydroxymethyl)1,3-propanediol (that is, tris $\cdot \mathrm{H}^{+}$) in water, has been described in detail in earlier papers $[3,5]$. The corresponding dissociation constant in $50 \mathrm{wt}$ percent methanol, referred not to the standard state in water but to that in this solvent, is termed $p\left({ }_{\mathrm{s}} K\right)$. The procedure for obtaining $p\left({ }_{s} K\right)$ is entirely analogous to that for the computation of $p K$ (that is, $p\left({ }_{w} K\right)$ ) in water.

"Apparent" values of $p\left({ }_{\mathrm{s}} K\right)^{\prime}$ were computed from the emf $E$ by the equation

$p\left({ }_{\mathrm{s}} K\right)^{\prime} \equiv p\left({ }_{\mathrm{s}} K\right)-\beta I=\frac{E-{ }_{\mathrm{s}} E^{\circ}}{(R T \ln 10) / F}+2 \log m_{1}-\log m_{2}$

$$
-\frac{2 A \sqrt{I}}{1+B a ̊ \sqrt{I}}
$$


TABLE 1. Electromotive force of the cell Pt; $\mathrm{H}_{2}(g, 1$ atm), tris $\mathrm{HCl}\left(\mathrm{m}_{1}\right)$, tris $\left(\mathrm{m}_{2}\right)$ in 50 wt \% methanol, $\mathrm{AgCl} ; \mathrm{Ag}$ from 10 to $40{ }^{\circ} \mathrm{C}$ (in volts)

Temperature, ${ }^{\circ} \mathrm{C}$

\begin{tabular}{l|l|l|l|l|l|l|l}
\hline \hline$m_{1}^{a}$ & 10 & 15 & 20 & 25 & 30 & 35 & 40 \\
\hline
\end{tabular}

Series I: $m_{2}=1.0080 m_{1}$

\begin{tabular}{|c|c|c|c|c|c|c|c|}
\hline 0.009554 & 0.78772 & 0.78553 & 0.78343 & & & & \\
\hline .009566 & & & & & 0.77857 & 0.77626 & 0.77394 \\
\hline .009575 & .78781 & .78567 & .78353 & .78099 & .77917 & .77681 & .77427 \\
\hline .01921 & .77366 & .77117 & .76898 & .76646 & .76365 & .76097 & .75839 \\
\hline .01922 & .77331 & & & & .76372 & .76092 & .75812 \\
\hline .02892 & .76635 & .76388 & .76135 & .75862 & & & \\
\hline .02892 & .76606 & .76357 & .76079 & .75830 & .75584 & .75298 & .75015 \\
\hline .03871 & .76056 & .75799 & .75538 & .75276 & .75001 & .74713 & .74414 \\
\hline .03872 & .76083 & .75830 & .75571 & .75299 & .75000 & .74712 & .74426 \\
\hline .04857 & .75609 & .75354 & .75086 & .74800 & & & \\
\hline .04857 & .75646 & .75384 & .75102 & .74829 & .74558 & .74263 & .73973 \\
\hline .04861 & .75624 & .75375 & .75106 & .74831 & .74564 & .74253 & .739 \\
\hline .05854 & .75322 & .75066 & .74787 & .74506 & .74205 & .73911 & .73607 \\
\hline .06849 & .75042 & .74777 & .74503 & .74222 & .73921 & .73616 & .73307 \\
\hline .06852 & .75031 & .74759 & .74482 & .74203 & .73915 & .73610 & .73311 \\
\hline .07867 & .74773 & .74499 & .74230 & .73941 & .73648 & .73340 & .73037 \\
\hline .07869 & .74789 & .74518 & .74239 & .73945 & .73630 & .73331 & .73017 \\
\hline .08882 & .74589 & .74313 & .74033 & .73745 & .73431 & .73121 & .72810 \\
\hline .09910 & .74397 & .74114 & .73840 & .73545 & .73255 & .72934 & .72618 \\
\hline
\end{tabular}

Series II: $m_{2}=1.0037 m_{1}$

\begin{tabular}{r|r|r|r|r|r|r|r}
\hline 0.01928 & 0.77339 & 0.77164 & 0.76912 & 0.76672 & 0.76381 & 0.76101 & 0.75826 \\
.03889 & .75992 & .75774 & .75545 & .75269 & .74992 & .74706 & .74417 \\
.05880 & .75248 & $\ldots \ldots \ldots \ldots \ldots$ & .74719 & .74464 & .74203 & .73906 & .73622 \\
.07900 & .74808 & .74532 & .74236 & .73958 & .73629 & .73321 & .73049 \\
.09948 & .74429 & .74149 & .73845 & .73564 & .73231 & .72925 & .72621 \\
\hline
\end{tabular}

Series III: $m_{2}=m_{1}$

\begin{tabular}{r|r|r|r|r|r|r|r}
\hline 0.01922 & 0.77405 & 0.77154 & 0.76925 & 0.76673 & 0.76415 & 0.76150 & 0.75856 \\
.03872 & .76077 & .75789 & .75541 & .75288 & .75020 & .74733 & .74421 \\
.05854 & .75304 & .75029 & .74774 & .74494 & .74200 & .73907 & .73595 \\
.07864 & .74796 & .74512 & .74242 & .73956 & .73656 & .73357 & .73044 \\
.09910 & .74371 & .74098 & .73829 & .73538 & .73230 & .72934 & .72610 \\
\hline
\end{tabular}

Series IV: $m_{2}=1.0022 m_{1}$

\begin{tabular}{c|r|r|r|r|r|r|r}
\hline 0.009576 & 0.78818 & 0.78571 & 0.78349 & 0.78128 & 0.77888 & 0.77649 & 0.77386 \\
.02894 & .76588 & .76331 & .76075 & .75825 & .75558 & .75273 & .74979 \\
.04857 & .75614 & .75357 & .75092 & .74824 & .74536 & .74239 & .73945 \\
.06853 & .75015 & .74743 & .74469 & .74189 & .73890 & .73579 & .73270 \\
.08890 & .74534 & .74261 & .73982 & .73700 & .73396 & .73089 & .72780 \\
\hline
\end{tabular}

${ }^{a}$ Tris hydrochloride was used to prepare the solutions of Series I and II, but hydrochloric acid was used for Series III and IV.

where $A$ and $B$ are constants of the Debye-Hückel theory $[6,7]$ and $\stackrel{\circ}{a}$ is the "ion-size parameter." The values of the standard emf ${ }_{s} E^{\circ}$ in 50 wt percent methanol have been reported elsewhere [4].

The acidic strength of tris hydrochloride in this solvent, as in water, falls in the region where solvolysis of both the free base and the protonated base is minimal; hence, the buffer ratio is accurately given by $m_{2} / m_{1}$, and the ionic strength $I$ is identical with $m_{1}$. The thermodynamic $p\left({ }_{s} K\right)$ is obtained by extrapolating values of $p\left({ }_{\mathrm{s}} K\right)^{\prime}$ to the limit of $I=0$ ("infinite dilution"). If the Debye-Hückel equation is an adequate representation of the activity-coefficient term, the plot of $p\left({ }_{\mathrm{s}} K\right)^{\prime}$ as a function of $I$ will be a straight line of slope $-\beta$ when a suitable value of the ion-size parameter $\stackrel{a}{a}$ is chosen. It will usually be curved for other values of $\stackrel{a}{a}$.

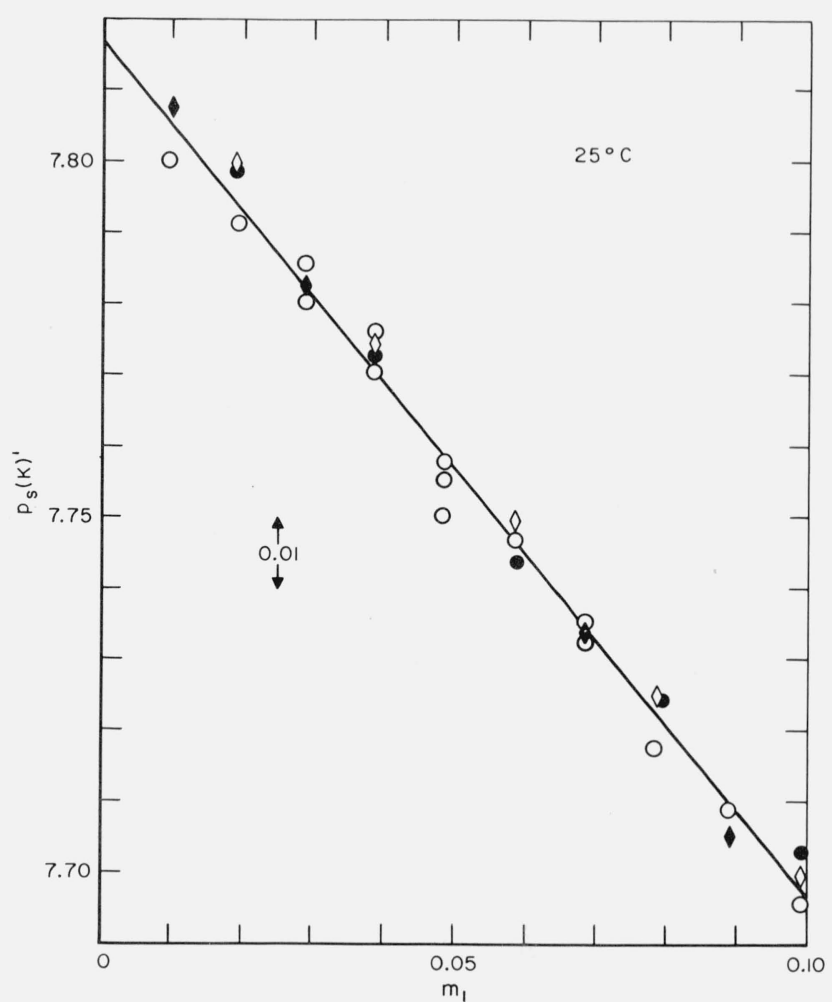

Figure 1. Plot of the apparent $\mathrm{p}\left({ }_{\mathrm{s}} \mathrm{K}\right)$ in 50-percent methanol as a function of $\mathrm{m}_{1}$ at $25^{\circ} \mathrm{C}$.

$$
\begin{aligned}
& \text { Series I. } \\
& \text { Series II. } \\
& \text { Series III. } \\
& \text { Series IV. }
\end{aligned}
$$

Figure 1 is a plot of the data at $25^{\circ} \mathrm{C}$. This straight line was obtained with a value of 0 for $\stackrel{\circ}{a}$ : it corresponds to a slope $-\beta=1.220$. The same value of $\stackrel{\circ}{a}$ proved suitable for the extrapolation at all temperatures from 10 to $40{ }^{\circ} \mathrm{C}$. The intercepts $p\left({ }_{\mathrm{s}} K\right)$ are summarized in table 2 , together with the standard deviations of the intercepts and the values of $p\left({ }_{\mathrm{w}} K\right)$ found in previous investigations $[3,5]$. These "observed" values of $p\left({ }_{\mathrm{s}} K\right)$ can be represented within about 0.001 unit by the following equation, which is of the form proposed by Harned and Robinson [8]:

$$
p\left({ }_{\mathrm{s}} K\right)=\frac{A_{1}}{T}-A_{2}+A_{3} T=\frac{3689.57}{T}-8.5466+0.013381 T
$$

TABLE 2. $\quad \mathrm{p}\left({ }_{\mathrm{S}} \mathrm{K}\right)$ values for the protonated form of tris in 50 wt percent methanol from 10 to $40{ }^{\circ} \mathrm{C}$

\begin{tabular}{c|c|r|r|r}
\hline \hline$t$ & $\left.p{ }_{\mathrm{s}} K\right)$ & \multicolumn{1}{|c|}{$\sigma_{\mathrm{i}}$} & $\begin{array}{c}p\left({ }_{\mathrm{S}} K\right) \\
\text { (calc.) }\end{array}$ & \multicolumn{1}{c}{$p\left({ }_{\mathrm{w}} K\right)$} \\
\hline${ }^{\circ} \mathrm{C}$ & & & & \\
10 & 8.273 & 0.002 & 8.273 & 8.516 \\
15 & 8.113 & .001 & 8.114 & 8.361 \\
20 & 7.962 & .001 & 7.962 & 8.213 \\
25 & 7.818 & .001 & 7.818 & 8.072 \\
30 & 7.681 & .001 & 7.681 & 7.934 \\
35 & 7.550 & .001 & 7.550 & 7.802 \\
40 & 7.426 & .001 & 7.426 & 7.676 \\
\end{tabular}


where $T$ is the temperature in ${ }^{\circ} \mathrm{K}$. The $p\left({ }_{s} K\right)$ calculated by eq (2) is given in the next to the last column of table 2.

It is evident that the protonated form of tris(hydroxymethyl)aminomethane is a stronger acid in $50 \mathrm{wt}$ percent methanol than it is in pure water, where the $p K$ is 8.072 at $25^{\circ} \mathrm{C}[3,5]$. This increase of strength is in accord with earlier measurements of the solvent effect of methanol on acids of this charge type $[9,10$, 11]. In general, the $p\left({ }_{s} K\right)$ goes through a minimum in the range of solvent compositions between 70 and 90 wt percent methanol and then rises sharply as the concentration of solvent water becomes low. It has been shown that this behavior cannot be explained by the electrostatic effects of the changing dielectric constant alone. Nevertheless, qualitative consistency among the solvent effects on $p K$ for acids of different charge types can be achieved if a second parameter characterizing the alteration in the basicity of the medium is introduced [12].

\section{Thermodynamic Constants for the Dissociation}

\subsection{Calculation of the Thermodynamic Quantities}

By the application of the usual thermodynamic formulas to eq (2), the changes of Gibbs energy, enthalpy, entropy, and heat capacity for the dissociation of the protonated form of tris in the standard state were calculated. The results from 10 to $40{ }^{\circ} \mathrm{C}$ are given by the equations

$$
\begin{aligned}
& \frac{\Delta G^{\circ}}{\mathrm{J} \mathrm{mol}^{-1}}=19.1445\left(A_{1}-A_{2} T+A_{3} T^{2}\right) \\
& \frac{\Delta H^{\circ}}{\mathrm{J} \mathrm{mol}^{-1}}=19.1445\left(A_{1}-A_{3} T^{2}\right) \\
& \frac{\Delta S^{\circ}}{\mathrm{J} \mathrm{mol}^{-1} \operatorname{deg}^{-1}}=19.1445\left(A_{2}-2 A_{3} T\right) \\
& \frac{\Delta C_{p}^{\circ}}{\mathrm{J} \mathrm{mol}^{-1} \mathrm{deg}^{-1}}=-19.1445\left(2 A_{3} T\right) .
\end{aligned}
$$

The values of these thermodynamic quantities in 50 wt percent methanol are compared in table 3 with the same quantities for the dissociation in the aqueous medium $[3,5]$. The standard deviations in 50-percent methanol can be estimated by the procedure outlined by Please [13]. Assuming a standard deviation of 0.001 in $p\left({ }_{\mathrm{s}} K\right)$, the results of the estimate are as follows:

\begin{tabular}{|c|c|c|c|}
\hline & In water & & In 50-percent \\
\hline $\begin{array}{l}\Delta G^{\circ} / \mathrm{kJ} \mathrm{mol}^{-1} \\
\Delta H^{\circ} / \mathrm{kJ} \mathrm{mol}^{-1} \\
\Delta S^{\circ} / \mathrm{J} \mathrm{mol}^{-1} \mathrm{deg}^{-1} \\
\Delta C_{p}^{\circ} / \mathrm{J} \mathrm{mol}^{-1} \mathrm{deg}^{-1}\end{array}$ & $\begin{array}{r}\text { Ref. [3] } \\
46.06(46.06) \\
47.80(47.41) \\
5.81(4.52) \\
-69.5(-91.4)\end{array}$ & $\begin{array}{r}46.07 \\
47.60 \\
5.11 \\
-63.6\end{array}$ & $\begin{array}{r}44.62 \\
47.86 \\
10.9 \\
-153\end{array}$ \\
\hline
\end{tabular}

$$
\begin{array}{lc}
\Delta G^{\circ}, 3 & \mathrm{~J} \mathrm{~mol}^{-1} \\
\Delta H^{\circ}, 64 & \mathrm{~J} \mathrm{~mol} \\
& \\
\Delta S^{\circ}, & 0.22 \mathrm{~J} \mathrm{~mol}^{-1} \mathrm{deg}^{-1} \\
\Delta C_{p}^{\circ}, 15 & \mathrm{~J} \mathrm{~mol}^{-1} \mathrm{deg}^{-1} .
\end{array}
$$

TABLE 3. Thermodynamic quantities for the acidic dissociation of the protonated form of tris at $25^{\circ} \mathrm{C}$

All of the values given were derived from a three-constant equation of the same form as eq (2) except those given in parentheses. In calculating these, $p K$ was fitted to a fourconstant equation (represented by eq (2) with an additional term $\mathrm{A}_{4} \mathrm{~T}^{2}$ added to the rightconstant equat
hand side).

The replacement of one-half of the water solvent, on a weight basis, by methanol has evidently failed to produce profound changes of a magnitude and character sufficient to suggest that the solvation pattern has been drastically altered. It seems likely, therefore, that the fundamental dissociation process in 50-percent methanol is, as in water, the transfer of a proton from combination with tris to combination with a basic water molecule.

\subsection{Significance of the Solvent Effect}

The effect of solvent on the Gibbs energy change for the dissociation of a weak acid has sometimes been ascribed to a change in the electrostatic selfenergy of the ions. The simplest expression for the electrostatic energy of a mole of univalent ions is that of Born [14]:

$$
G_{\mathrm{el}}=\frac{N e^{2}}{2} \frac{1}{\epsilon r_{\mathrm{s}}}
$$

where $N$ is the Avogadro number, $e$ the electronic charge, $\epsilon$ the dielectric constant of the medium, and $r_{\mathrm{s}}$ the radius of the (spherical) ion. The assumption made here is that the solvent is a continuous medium with a dielectric constant equal at all points to the macroscopic dielectric constant.

The dissociation of tris ${ }^{\cdot} \mathrm{H}^{+}$is an isoelectric process:

$$
\mathrm{BH}^{+}+\mathrm{SH}=\mathrm{B}+\mathrm{SH}_{2}^{+}
$$

where $\mathrm{BH}^{+}$represents the protonated base and $\mathrm{SH}$ is the amphiprotic solvent. The change in electrostatic energy on transfer from water to 50-percent methanol is then

$$
\Delta G_{\mathrm{el}}=\frac{N e^{2}}{2}\left(\frac{1}{\epsilon^{\prime}}-\frac{1}{\epsilon}\right)\left(\frac{1}{r_{\mathrm{H}^{+}}}-\frac{1}{r_{\mathrm{BH}^{+}}}\right)
$$

where $\epsilon^{\prime}$ is the dielectric constant of 50 -percent methanol $\left(56.3\right.$ at $\left.25{ }^{\circ} \mathrm{C}\right)$ and $\epsilon$ is that of water $(78.30$ at 25 $\left.{ }^{\circ} \mathrm{C}\right)$. Thus $\Delta G_{\mathrm{el}}$ will have a positive value if $r_{\mathrm{BH}^{+}}>r_{\mathrm{H}^{+}}$. Although the value to be assigned to the radius of the hydrogen ion (designated $\mathrm{SH}_{2}^{+}$, or simply $\mathrm{H}^{+}$in the absence of precise knowledge of its solvated structure) may be in dispute, it is difficult to see how this radius could be larger than that of the protonated tris cation, $\mathrm{BH}^{+}$. 
An atom model of the tris cation shows that this ion is not unlike the tetraethylammonium ion in size and shape. A radius of $4 \AA$ has been ascribed to the latter [6], and it may be taken as a reasonable estimate of the radius of the tris cation as well. An ion with this radius contributes $0.87 \mathrm{~kJ} \mathrm{~mol}^{-1}$ to the electrostatic energy change on transfer from water to 50-percent methanol.

The difficulty in applying eq (8) to an acidic dissociation process lies in our ignorance of the effective radius of the hydrogen ion. There is, however, considerable evidence [15] that the hydrogen ion is intimately associated with four water molecules in solvents containing a considerable amount of water, and we can reasonably identify the radius of this hydrated hydrogen ion with the diameter of the water molecule, $2.8 \AA$. An ion of this size contributes $1.24 \mathrm{~kJ} \mathrm{~mol}^{-1}$ to the electrostatic energy.

The total electrostatic effect on dissociation of the protonated form of tris should therefore be $0.4 \mathrm{~kJ} \mathrm{~mol}^{-1}$. The experimental value of $-1.45 \mathrm{~kJ} \mathrm{~mol}^{-1}$ is very different and of opposite sign. A similar anomaly seems to hold for all protonated bases, for example o-chloroanilinium ion and $m$-nitroanilinium ion [12]. This result has been ascribed to an increase in the basicity of the solvent on the addition of methanol, which may well be due to a degradation of the water structure by methanol. A structure-promoting entity is more effective in a methanolic solvent than in water, because there are more opportunities for the water structure to be promoted.

Hydrogen ion should be one of the best structure promoters, and therefore we have a reasonable explanation of the increase in acidity of protonated tris on addition of methanol. The magnitude of the effect is surprising, however; it must be of the order of -2 $\mathrm{kJ} \mathrm{mol}^{-1}$ if the total effect is $-1.45 \mathrm{~kJ} \mathrm{~mol}^{-1}$ and +0.4 $\mathrm{kJ} \mathrm{mol}^{-1}$ must be ascribed to the electrostatic effect.

It is worthwhile to consider whether this discrepancy may be due to inadequacies of the simple Born treatment. In an effort to explain the Gibbs energy changes on the transfer of electrolytes from water to deuterium oxide, Hepler [16] has recently considered the consequences of assuming a model suggested by the work of Ritson and Hasted [17]. Three regions of solvent distribution around an ion are distinguished in this treatment. From the surface of the ion of radius $r_{\mathrm{s}}$ to a distance $1.5 \AA$ from its center is a region of dielectric saturation, with a dielectric constant $\epsilon_{\text {sat }}$. For water solvents, $\epsilon_{\text {sat }}$ may be taken as 5 [16]. At distances $(r)$ greater than $4 \AA$ from the center of the ion, the solvent has its macroscopic dielectric constant $\epsilon_{0}$. In the intermediate region, the dielectric constant varies linearly with $r$ :

$$
\epsilon=\frac{\epsilon_{0}-\epsilon_{\mathrm{sat}}}{2.5}(r-1.5)+\epsilon_{\mathrm{sat}} .
$$

The variation of the dielectric constant in water and in 50-percent methanol with distance from the center of the ion is shown schematically in figure 2 .
The electrostatic energy of a univalent ion is then given by

$$
G_{\mathrm{el}}=\frac{e^{2}}{2}\left\{\int_{r_{\mathrm{s}}}^{1.5} \frac{d r}{\epsilon_{\mathrm{sat}} r^{2}}+\int_{1.5}^{4} \frac{d r}{(X r-Y) r^{2}}+\int_{4}^{\infty} \frac{d r}{\epsilon_{0} r^{2}}\right\}
$$

where $X=0.4\left(\epsilon_{0}-\epsilon_{\text {sat }}\right)$. and $Y=1.5 X-\epsilon_{\text {sat. }}$ Upon integration we obtain

$$
\begin{array}{r}
G_{\mathrm{el}}=\frac{e^{2}}{2}\left\{\frac{1}{\epsilon_{\mathrm{sat}}}\left(\frac{1}{r_{\mathrm{s}}}-\frac{1}{1.5}\right)-\frac{0.417}{Y}+\frac{X}{Y^{2}} \ln \frac{0.375 \epsilon_{0}}{\epsilon_{\mathrm{sat}}}\right. \\
\left.+\frac{0.25}{\epsilon_{0}}\right\} .
\end{array}
$$

For the transfer of a mole of ions from water to another solvent (identified by a prime mark), we have

$$
\begin{aligned}
& \Delta G_{\mathrm{el}}=\frac{N e^{2}}{2}\left\{\left(\frac{1}{\epsilon_{\mathrm{sat}}^{\prime}}-\frac{1}{\epsilon_{\mathrm{sat}}}\right)\left(\frac{1}{r_{\mathrm{s}}}-\frac{1}{1.5}\right)-0.417\left(\frac{1}{Y^{\prime}}-\frac{1}{Y}\right)\right. \\
& \left.+\frac{X^{\prime}}{\left(Y^{\prime}\right)^{2}} \ln \frac{0.375 \epsilon_{0}^{\prime}}{\epsilon_{\mathrm{sat}}^{\prime}}-\frac{X}{Y^{2}} \ln \frac{0.375 \epsilon_{0}}{\epsilon_{\mathrm{sat}}}+0.25\left(\frac{1}{\epsilon_{0}^{\prime}}-\frac{1}{\epsilon_{0}}\right)\right\} .
\end{aligned}
$$

For an ion of radius $4 \AA$, only the last term of eq (10) appears, and the electrostatic energy of the ion is identical with that calculated by the Born equation. Thus the electrostatic energy $\left(\Delta G_{\mathrm{BH}^{+}}\right)$of the protonated tris cation is still $0.87 \mathrm{~kJ} \mathrm{~mol}^{-1}$. If the hydrogen ion has a radius of $2.8 \AA$, the first term of eq (10) disappears and the integration of the second term is to be made

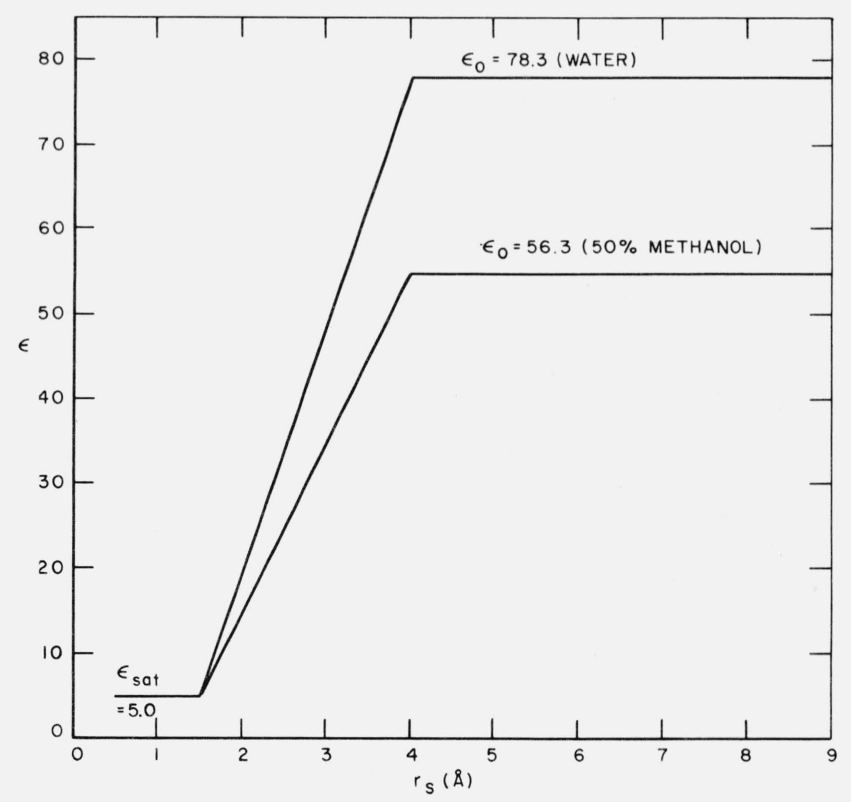

FIGURE 2. Variation of the dielectric constant of water and 50 . percent aqueous methanol in the vicinity of an ion; $\mathrm{r}_{\mathrm{s}}$ is the distance from the center of the ion. 
from 2.8 to $4 \AA$. Equation (12) now becomes

$$
\begin{array}{r}
\Delta G_{\mathrm{el}}=\frac{N e^{2}}{2}\left\{-0.107\left(\frac{1}{Y^{\prime}}-\frac{1}{Y}\right)+\frac{X^{\prime}}{\left(Y^{\prime}\right)^{2}} \ln \frac{0.7 \epsilon_{0}^{\prime}}{2.8 X^{\prime}-Y^{\prime}}\right. \\
\left.-\frac{X}{Y^{2}} \ln \frac{0.7 \epsilon_{0}}{2.8 X-Y}+0.25\left(\frac{1}{\epsilon_{0}^{\prime}}-\frac{1}{\epsilon_{0}}\right)\right\}
\end{array}
$$

for the hydrogen ion. On substitution of the appropriate values of $\epsilon_{0}$ and $\epsilon_{0}^{\prime}$ we obtain $\Delta G_{\mathrm{H}+}=1.35 \mathrm{~kJ}$ $\mathrm{mol}^{-1}$ for the transfer of a mole hydrogen ions from water to 50-percent methanol.

It thus appears that the calculated gain in electrostatic energy due to dissociation of the tris cation is now $+0.48 \mathrm{~kJ} \mathrm{~mol}^{-1}$, in even more marked contrast with the observed value of $-1.45 \mathrm{~kJ} \mathrm{~mol}^{-1}$. The calculation therefore serves to emphasize that, no matter how doubtful some of the assumptions about the ionic radii may be, the basicity effect in the opposite direction must be of considerable magnitude, in fact about $2 \mathrm{~kJ} \mathrm{~mol}^{-1}$.

Further evidence for the existence of a pronounced increase in solvent basicity when methanol is added to water to make a $50 \mathrm{wt}$ percent mixture is found in the energies of transfer of hydrochloric acid from water to this mixed solvent. The standard emf of the cell with hydrogen and silver-silver chloride electrodes in 50percent methanol has been found [4] to be $0.19058 \mathrm{~V}$ at $25^{\circ} \mathrm{C}$, or $31.76 \mathrm{mV}$ lower than the value in water. Thus the transfer of a mole of hydrogen ions and a mole of chloride ions from water to 50-percent methanol is accompanied by an increase in Gibbs energy of $3.06 \mathrm{~kJ}$. The radius of the chloride ion is known [18] to be $1.81 \AA$. Using the Born model, we calculate that the chloride ion should contribute $1.91 \mathrm{~kJ} \mathrm{~mol}^{-1}$ which, together with $1.24 \mathrm{~kJ} \mathrm{~mol}^{-1}$ for the hydrogen ion, gives $3.15 \mathrm{~kJ} \mathrm{~mol}^{-1}$ for the transfer of hydrochloric acid.

This calculated figure is perhaps not in very serious disagreement with the observed value. Nevertheless, it is derived on the assumption that the macroscopic dielectric constant holds at a distance of $1.81 \AA$. It is in this region that the Hepler treatment gives results markedly different from those furnished by the Born equation. For example, the electrostatic term for the chloride ion is $3.08 \mathrm{~kJ} \mathrm{~mol}^{-1}$ by the Hepler treatment. This value, together with $1.35 \mathrm{~kJ} \mathrm{~mol}^{-1}$ for the hydrogen ion, gives a total of $4.43 \mathrm{~kJ} \mathrm{~mol}^{-1}$ for the transfer process from electrostatic considerations alone. By comparison with the observed Gibbs energy change $\left(3.06 \mathrm{~kJ} \mathrm{~mol}^{-1}\right)$ it is again seen that the basicity effect must amount to about $1.4 \mathrm{~kJ} \mathrm{~mol}^{-1}$.

Data are available for the solvent effect on the dissociation of acetic acid, but, in view of the very unsymmetrical shape of the acetate anion, it is doubtful that either the Born equation or the Hepler treatment can be usefully applied to data for this acid. However, the second dissociation of phosphoric acid is a more suitable case, because of the higher symmetry of the oxygen atoms surrounding the phosphorus atom.
From density data [19], the partial molal volume of potassium dihydrogen phosphate can be estimated to be $39 \mathrm{ml} \mathrm{mol}^{-1}$; a value of $1.5 \mathrm{ml} \mathrm{mol}^{-1}$ has been ascribed to the potassium ion [20], leaving $37.5 \mathrm{ml}$ $\mathrm{mol}^{-1}$ for the dihydrogen phosphate ion. A mole of spherical ions with radii $2.5 \AA$ would have this partial molal volume and would contribute $1.64 \mathrm{~kJ} \mathrm{~mol}^{-1}$ to the electrostatic energy.

Similar calculations are difficult to make for the bivalent hydrogen phosphate ion $\left(\mathrm{HPO}_{4}^{=}\right)$, because the density data for sodium dihydrogen phosphate solutions are fragmentary. The available data, however, suggest a radius of about $2 \AA$ and, therefore, a contribution of $2.49 \mathrm{~kJ} \mathrm{~mol}^{-1}$ to the electrostatic energy. This figure must be quadrupled, however, because of the double charge on this ion. The net effect due to hydrogen ion, $\mathrm{H}_{2} \mathrm{PO}_{4}^{-}$, and $\mathrm{HPO}_{4}^{-}$is $9.7 \mathrm{~kJ} \mathrm{~mol}^{-1}$ compared with the observed value of $7.1 \mathrm{~kJ} \mathrm{~mol}^{-1}$.

It would be unwise to imply that these values of the electrostatic energy calculated with only estimated values of the ionic radii have any exactitude. It is important, however, to note that whenever a reasonable assumption can be made about these ionic radii, the electrostatic contribution is higher by 1 to $3 \mathrm{~kJ}$ than the observed energy change. In other words, there is always a term of considerable magnitude for the "basicity effect" that does not figure in the electrostatic treatment.

\section{Activity Coefficient of Tris Hydrochloride}

The success of the extrapolation procedure embodied in eq (1) in this and other similar situations ${ }^{6}$ justifies the substitution of the Debye-Hückel formula for the activity-coefficient term in the expression for the dissociation constant of a protonated amine, $\mathrm{BH}^{+}$. Thus for the process

$$
\mathrm{BH}^{+}+\mathrm{SH}=\mathrm{B}+\mathrm{SH}_{2}^{+}
$$

one can write [23]

$$
\log \gamma_{ \pm}^{\prime} \equiv \log \gamma_{ \pm}\left(\frac{a_{\mathrm{SH}}}{\gamma_{\mathrm{B}}}\right)^{1 / 2}=\frac{-A \sqrt{ } I}{1+B \stackrel{\circ}{ } I}+0.5 \beta I
$$

where $\gamma_{ \pm}$is the mean activity coefficient of the amine hydrochloride.

Straight-line extrapolations such as those shown in figure 1 could not be obtained when "reasonable" values of 3 to $5 \AA$ were used for the ion-size parameter $\stackrel{\circ}{a}$ in eq (1), and the line shown corresponds to $\stackrel{\circ}{a}=0$. A similar anomalous behavior of the activity-coefficient term in aqueous buffer solutions composed of other aliphatic or aromatic amines and their salts has been observed in the course of a number of similar studies. These investigations have dealt with ammonia [23], tris [5], 2-methyl-2-amino-propanediol [24], $t$-butylamine [22], ethanolamine [21], diethanolamine [25],

\footnotetext{
${ }^{6}$ See, for example, measurements on ethanolamine [21] and $t$-butylamine [22]
} 
triethanolamine [26], aminopyridine [27], and piperidine [28]. The abnormally small or even negative value of $\stackrel{\circ}{a}$ suggests that ion pairs of moderate stability exist in these buffer solutions, and in the study of piperidine it was possible to account for the results with an ion size of $4 \AA$ when a correction was made for ion-pair formation [28].

These observations are surprising in view of the fact that the activity coefficient of aqueous ammonium chloride at $0.1 \mathrm{~m}$ is not greatly different from that of potassium chloride at the same molality [29]. The isopiestic method by which these activity coefficients were determined is unfortunately not capable of furnishing precise data for activity coefficients much below a molality of 0.1 .

One can, however, derive the activity coefficients of ammonium chloride and tris hydrochloride in equimolal buffer solutions $\left[\mathrm{BH}^{+}(m), \mathrm{B}(m)\right]$ from the emf data used to derive $p K$ and from the slopes of the extrapolation lines such as those shown in figure 1 (see eq (15)). The results for the activity coefficients and osmotic coefficients of ammonium chloride and tris hydrochloride are compared in table 4 with the corresponding values for potassium chloride [30]. It is apparent that no great difference exists in dilute aqueous solutions. The value $(0.758)$ found in this 'way for the mean activity coefficient of tris hydrochloride $(0.1 \mathrm{~m})$ in the presence of tris $(0.1 \mathrm{~m})$ is in good agreement with the value $(0.770)$ found by isopiestic measurements [31] of $0.1 \mathrm{~m}$ tris hydrochloride without any added tris.

The Hückel equation utilized in eq (1) describes the activity coeis ient term by two parameters in two separate terms. The results shown in table 4 mean, therefore, that the variation of the activity coefficient of ammonium chloride (and probably of amine hydrochlorides as well) with ionic strength is partitioned between the two terms in a different manner from that characterizing the behavior of the alkali metal chlorides. The reason for this difference should probably be sought in the different structures of the hydration shells of the two types of cation and in the concomitant effects on the microscopic dielectric constant in the immediate vicinity of the ions.

TABLE 4. Comparison of the activity coefficients and osmotic coefficients of $\mathrm{KCl}, \mathrm{NH}_{4} \mathrm{Cl}$, and tris. $\mathrm{HCl}$ in aqueous solutions at $25^{\circ} \mathrm{C}$.

\begin{tabular}{|c|c|c|c|c|c|c|}
\hline \multirow{2}{*}{$m$} & \multicolumn{3}{|c|}{$\gamma \pm$ for -} & \multicolumn{3}{|c|}{$\phi$ for -} \\
\hline & $\mathrm{KCl}$ & $\mathrm{NH}_{4} \mathrm{Cl}$ & Tris $\cdot \mathrm{HCl}$ & $\mathrm{KCl}$ & $\mathrm{NH}_{4} \mathrm{Cl}$ & Tris $\cdot \mathrm{HCl}$ \\
\hline 0.005 & 0.928 & 0.925 & 0.925 & 0.977 & 0.975 & 0.975 \\
\hline .01 & .902 & .899 & .898 & .968 & .966 & .966 \\
\hline .02 & .870 & .865 & .864 & .957 & .955 & .954 \\
\hline .05 & .817 & .809 & .808 & .941 & .937 & .936 \\
\hline .07 & .795 & .786 & .785 & .934 & .931 & .930 \\
\hline .1 & .770 & .760 & .758 & .927 & .923 & .923 \\
\hline
\end{tabular}

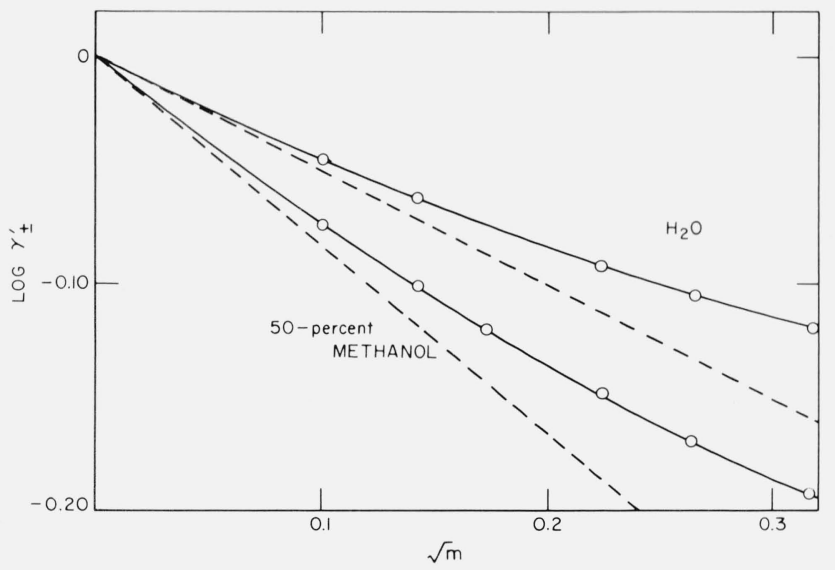

Figure 3. Comparison of $\log \gamma_{ \pm}^{\prime}(e q(15))$ in the two solvents water and 50-percent methanol.

The dashed lines are the Debye-Hückel limiting slopes.

The data for tris buffers in 50 wt percent methanol cannot be used to derive the activity coefficients for tris hydrochloride by eq (15), in the absence of information concerning the activities of both tris and the solvent in the methanolic medium. It is of interest, however, to compare the left-hand side of eq (15) for equimolal buffers of tris and its hydrochloride $\left(m_{1}=m_{2}\right)$ in 50 wt percent methanol with that for the same buffers in water. Such a comparison is shown in figure 3. The standard states in the two solvents differ, so that the activity coefficient becomes unity at $\mathrm{I}=0$ in each case. The Debye-Hückel limiting slopes for the two solvents are indicated by dashed lines, and the general similarity of the curves suggests that there are no great differences in the pattern of behavior in the two media of different compositions. As expected, departures from ideality are greater, at a given ionic strength, in the solvent of lower dielectric constant. Furthermore, the positive deviations from the limiting law are more pronounced in 50-percent methanol than in water.

Although this behavior is a consequence of a larger value of $-\beta$ (eq (15)) in the methanolic solvent, it is impossible at the present time to state with certainty whether it arises from differences in the salt effect on $\gamma_{ \pm}$or on $\left(a_{\mathrm{SH}} / \gamma_{\mathrm{B}}\right)$. It is likewise impossible to decide on the basis of this evidence what is the true nature of $\mathrm{SH}$ in eq (14), that is, which of the two types of solvent molecules plays the predominant role in the protolysis of $\mathrm{BH}^{+}$. Here again, there seems to be little evidence of a drastic change in the nature of the reaction process, such as would be expected if methanol replaced water to any large extent in the solvation shells of the proton and the acid $\mathrm{BH}^{+}$or base B. 


\section{References}

[1] M. Paabo, R. A. Robinson, and R. G. Bates, J. Am. Chem. Soc. 87, 415 (1965).

[2] R. G. Bates, Ann. N. Y. Acad. Sci. 92, 341 (1961).

[3] S. P. Datta, A. K. Grzybowski, and B. A. Wilson, J. Chem. Soc. (London), 792 (1963).

[4] M. Paabo, R. A. Robinson, and R. G. Bates, J. Chem. Eng. Data 9, 374 (1964).

[5] R. G. Bates and H. B. Hetzer, J. Phys. Chem. 65, 667 (1961).

[6] R. A. Robinson and R. H. Stokes, Electrolyte Solutions, 2d ed., p. 125 and app. 8.1 (Butterworths Scientific Publications, London, 1959).

[7] R. G. Bates, Determination of $p \mathrm{H}$, p. 406 (John Wiley \& Sons, Inc., New York, N.Y., 1964).

[8] H. S. Harned and R. A. Robinson, Trans. Faraday Soc. 36, 973 (1940).

[9] A. L. Bacarella, E. Grunwald, H. P. Marshall, and E. L. Purlee, J. Org. Chem. 20, 747 (1955).

[10] C. L. deLigny, H. Loriaux, and A. Ruiter, Rec. Trav. Chim. 80, 727 (1961).

[11] D. Rosenthal, H. B. Hetzer, and R. G. Bates, J. Am. Chem. Soc. 86, 549 (1964).

[12] E. E. Sager, R. A. Robinson, and R. G. Bates, J. Res. NBS 68A (Phys. and Chem.) No. 3, 305 (1964).

[13] N. W. Please, Biochem. J. 56, 196 (1954).

[14] M. Born, Z. Physik 1, 45, 221 (1920).

[15] R. P. Bell and K. N. Bascombe, Disc. Faraday Soc. 34, 158 (1957).
[16] L. G. Hepler, Aust. J. Chem. 1 7, 587 (1964).

[17] D. M. Ritson and J. B. Hasted, J. Chem. Phys. 16, 11 (1948).

[18] L. Pauling, The nature of the chemical bond, ch. X (Cornell University Press, Ithaca, N.Y., 1940).

[19] International Critical Tables, Vol. 3, p. 80 (McGraw-Hill Book Co., Inc., New York, N.Y., 1928).

[20] R. A. Robinson and R. H. Stokes, Trans. Faraday Soc. 53, 301 (1957).

[21] R. G. Bates and G. D. Pinching, J. Res. NBS 46, 349 (1951) RP2205.

[22] H. B. Hetzer, R. A. Robinson, and R. G. Bates, J. Phys. Chem. 66, 2696 (1962)

[23] R. G. Bates and G. D. Pinching, J. Res. NBS 42, 419 (1949) RP1982.

[24] H. B. Hetzer and R. G. Bates, J. Phys. Chem. 66, 308 (1962).

[25] V. E. Bower, R. A. Robinson, and R. G. Bates, J. Res. NBS 66A (Phys. and Chem.), No. 1, 71 (1962).

[26] R. G. Bates and G. F. Allen, J. Res. NBS 64A (Phys. and Chem.), No. 4, 343 (1960).

[27] R. G. Bates and H. B. Hetzer, J. Res. NBS 64A (Phys. and Chem.), No. 5, 427 (1960).

[28] R. G. Bates and V. E. Bower, J. Res. NBS 57, 153 (1956) RP2705.

[29] B. F. Wishaw and R. H. Stokes, Trans. Faraday Soc. 49, 27 (1953).

[30] W. J. Hornibrook, G. J. Janz, and A. R. Gordon, J. Am. Chem. Soc. 64, $513(1942)$.

[31] R. A. Robinson and V. E. Bower, J. Chem. Eng. Data, in press.

(Paper 69A3-345) 